\title{
Bistable photonic nanostructures based on molecular spin crossover complexes
}

\author{
Gábor Molnár*a , Il'ya A. Gural'skiy ${ }^{\mathrm{a}, \mathrm{b}}$, Lionel Salmon ${ }^{\mathrm{a}}$, William Nicolazzi ${ }^{\mathrm{a}}$, Carlos Quintero ${ }^{\mathrm{a}}$, Amal \\ Akou $^{\mathrm{a}}$, Khaldoun Abdul-kader ${ }^{\mathrm{a}}$, Gautier Félix ${ }^{\mathrm{a}}$, Tarik Mahfoud ${ }^{\mathrm{a}, \mathrm{c}}$, Christian Bergaud ${ }^{\mathrm{d}}$, Carlos \\ Bartual-Murgui $^{\text {a,d }}$, Christophe Thibault ${ }^{\mathrm{d}}$, Christophe Vieu $^{\mathrm{d}}$, Azzedine Bousseksou $^{\mathrm{a}}$ \\ ${ }^{a}$ Laboratoire de Chimie de Coordination, CNRS \& Université de Toulouse (UPS, INPT), 205 route \\ de Narbonne, F-31077 Toulouse, France; \\ ${ }^{\mathrm{b}}$ Department of Chemistry, National Taras Shevchenko University, 62 Vladimirska str., $01601 \mathrm{Kiev}$, \\ Ukraine \\ ${ }^{c}$ Optics \& Photonics Center (OPC), Moroccan Foundation for Science, Innovation and Research \\ (MAScIR); Technopolis Rabatshore, Morocco \\ ${ }^{d}$ LAAS, CNRS \& Université de Toulouse (UPS, INSA, IAES), 7 avenue du colonel Roche, 31077 \\ Toulouse, France
}

*gabor.molnar@lcc-toulouse.fr; phone +33(0)561333190; fax +33(0)561553003; www.lcc-toulouse.fr

\begin{abstract}
In this paper we discuss the elaboration, optical properties and possible applications of thin films and nano-patterns of molecular spin crossover complexes. These bistable nanostructures can respond reversibly with fast response times to various external stimuli, such as temperature changes, application of an external pressure, light irradiation or exposure to gas/vapor molecules. The response can be either transient (gating) or non-volatile (switching) depending on the experimental conditions. We show that these assets provide a very appealing scope for a variety of applications including tunable photonic devices, thermal imaging and chemical sensors. In particular, we discuss three photonic application principles based on fluorescence energy transfer, grating diffraction and guided plasmon-polariton waves.
\end{abstract}

Keywords: molecular spin crossover, bistability, nano-patterning, fluorescence, diffraction gratings, surface plasmons

\section{INTRODUCTION}

Spin crossover (SCO) complexes of $3 d^{4}-3 d^{7}$ transition metal ions $\left(\mathrm{Cr}^{\mathrm{II}}, \mathrm{Mn}^{\mathrm{II}}, \mathrm{Mn}^{\mathrm{III}}, \mathrm{Co}^{\mathrm{II}}, \mathrm{Co}^{\mathrm{III}}, \mathrm{Fe}^{\mathrm{II}}, \mathrm{Fe}^{\mathrm{III}}\right)$ represent an important class of bistable materials for which switching between high-spin (HS) and low-spin (LS) electronic configurations can be obtained by diverse external stimuli such as temperature, pressure, light irradiation, magnetic fields or even the adsorption of gas/vapor molecules. ${ }^{1}$ The switching of molecular spin-states is accompanied with a spectacular change of various physical properties. For this reason the possible applications of these materials, including information storage, display and switching devices, pigments and sensors continue to draw much attention. Up to now, efforts have been focused primarily on color changes for developing practical applications. ${ }^{2}$ In a few cases, the absorbance changes were exploited through luminescence doping, where the basic idea is to use a luminophore, whose luminescence is selectively quenched in a given spin state of the metal ions in the material due to a radiative or resonant energy transfer process. ${ }^{3-10}$ Refractive index changes associated with the $\mathrm{SCO}\left(\Delta n_{H L}=n_{H S}-n_{L S}\right.$, where $n$ is the real part of the complex refractive index $n^{*}=n+i k$ ) provide also interesting prospects for a range of photonic applications. The relevance of this property has been less widely recognized, probably due to the lack of suitable thin films and photonic structures exhibiting SCO. Indeed, previous studies in this direction used mainly bulk samples, ${ }^{11-14}$ which are not convenient for most applications and make for difficulty also to obtain accurate refractive index data in the two spin states. Recently, however, considerable progress has been made in the synthesis of SCO nano-materials as patterned or continuous thin films, nano-composites or nanoparticles. ${ }^{15}$ These new nano-materials allowed us to determine with accuracy the refractive index changes accompanying the spin crossover by a variety of standard photonics methods, such as spectroscopic ellipsometry, grating diffraction or surface plasmon resonance spectroscopy. ${ }^{16-17}$ In the present paper we 
will discuss the basic optical properties of molecular spin crossover materials and describe their possible applications in tunable photonic devices.

\section{OPTICAL ABSORPTION AND LUMINESCENT DOPING}

\subsection{Origin of the color change accompanying the spin crossover}

The change of the spin state of the molecule leads naturally to a complete change of its electronic absorption spectra. For example, in the case of an octahedral complex with $3 d^{6}$ electronic configuration $\left(\mathrm{Fe}^{\mathrm{II}}, \mathrm{Co}^{\mathrm{III}}\right)$ the ground state of the molecule may change from the singlet $\left({ }^{1} \mathrm{~A}_{1 \mathrm{~g}}\right) \mathrm{LS}$ to the quintet $\left({ }^{5} \mathrm{~T}_{2 \mathrm{~g}}\right) \mathrm{HS}$ electronic configuration (figure 1). From the Tanabe-Sugano diagram it appears clearly that in the HS state there is only one spin-allowed, metal-centered $d-d$ transition $\left({ }^{5} \mathrm{~T}_{2} \rightarrow{ }^{5} \mathrm{E}\right)$, while in the LS state there are two transitions of this type $\left({ }^{1} \mathrm{~A}_{1} \rightarrow{ }^{1} \mathrm{~T}_{1},{ }^{1} \mathrm{~A}_{1} \rightarrow{ }^{1} \mathrm{~T}_{2}\right)$. In addition, weak spin-forbidden transitions $\left({ }^{1} \mathrm{~A}_{1} \rightarrow{ }^{3} \mathrm{~T}_{1},{ }^{1} \mathrm{~A}_{1} \rightarrow{ }^{3} \mathrm{~T}_{2}\right)$ can be also detected in the LS spectra. Since the spin crossover phenomenon occurs for a relatively narrow range of ligand field strengths, the visible absorption spectra of different SCO complexes with the same metal ion and the same donor atoms (e.g. $\mathrm{Fe}^{\mathrm{II}} \mathrm{N}_{6}$ ) is expected to be fairly similar. In reality, however, the color of the compounds can be rather different due to the occurrence of various charge transfer transitions in the visible range. In particular, metal-ligand charge transfer (MLCT) bands are often observed for aromatic ligands with low-lying $\pi *$ orbitals. Charge transfer transitions involving molecular orbitals located primarily on the metal ion will be inevitably altered by the spin state of this latter. Since these transitions are usually very intense when compared to the relatively weak (Laporte forbidden) $d$ - $d$ ligand-field transitions they can completely dominate the perceptible color of the sample.
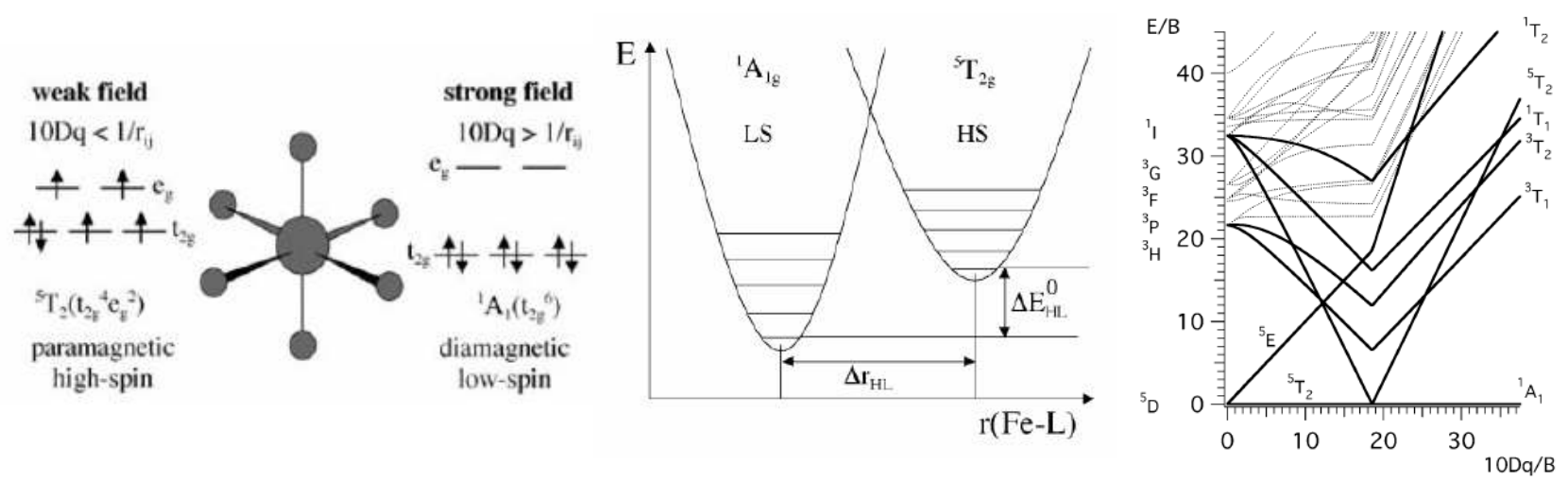

Figure 1. (left panel) High spin (weak ligand field) and low spin (strong ligand field) electronic configurations of a $3 d^{6}$ ion $\left(\mathrm{Fe}^{\mathrm{II}}, \mathrm{Co}{ }^{\mathrm{III}}\right)$ in an octahedral coordination environment. (middle panel) Potential energy of the high spin ${ }^{5} \mathrm{~T}_{2 \mathrm{~g}}$ ) and low spin $\left({ }^{1} \mathrm{~A}_{1 \mathrm{~g}}\right)$ states along the totally symmetric metal - ligand stretching coordinate in the harmonic approximation. (right panel) Tanabe-Sugano diagram for a transition metal ion with six $d$ electrons. Adapted from ref. [18].

As an example figure 2 shows the optical absorption spectra of the spin crossover complex $\left[\mathrm{Fe}^{\mathrm{II}}(\mathrm{hptrz})_{3}\right](\mathrm{OTs})_{2}$ in the visible wavelength range (where hptrz and OTs stand for 4-heptyl-1,2,4-triazole and tosylate, respectively). In the high temperature (HS) state the sample is nearly transparent. (Actually, it displays only a weak, broad absorption in the near infrared range, which can be assigned to the ${ }^{5} \mathrm{~T}_{2} \rightarrow{ }^{5} \mathrm{E}$ transition.) On the other hand, in the low temperature (LS) state the sample displays a light violet color due to an absorption band centered at $\sim 540 \mathrm{~nm}$, which can be assigned to the ${ }^{1} \mathrm{~A}_{1} \rightarrow{ }^{1} \mathrm{~T}_{1}$ transition. One can observe also the onset of a very intense charge transfer band below $450 \mathrm{~nm}$ in the LS state.

\subsection{Luminescent doping}

For many applications detection of transmitted or reflected/scattered light intensity changes is not convenient. On the other hand, fluorescence provides often better sensitivity and contrast, resulting in high signal-to-noise ratios for detection methods. If the emission and/or excitation spectrum of a luminophore shows significant overlap with the HS absorption band (but not with the LS absorption band) of the SCO material, the luminescent response can be quenched in the HS state. If the overlap is with the LS absorption band only, then the reverse is true. Hence, through the judicious choice of the luminophore, it should be possible to modulate luminescent properties as a function of the spin state of the SCO material. One shall note that beside the close spectral overlap, there are many other constraints to be taken into 
account, such as low photobleaching and high energy transfer rate. This latter can be achieved only if the separation between the metal ion and the luminophore is kept short (i.e. less than a few $\mathrm{nm}$ ).
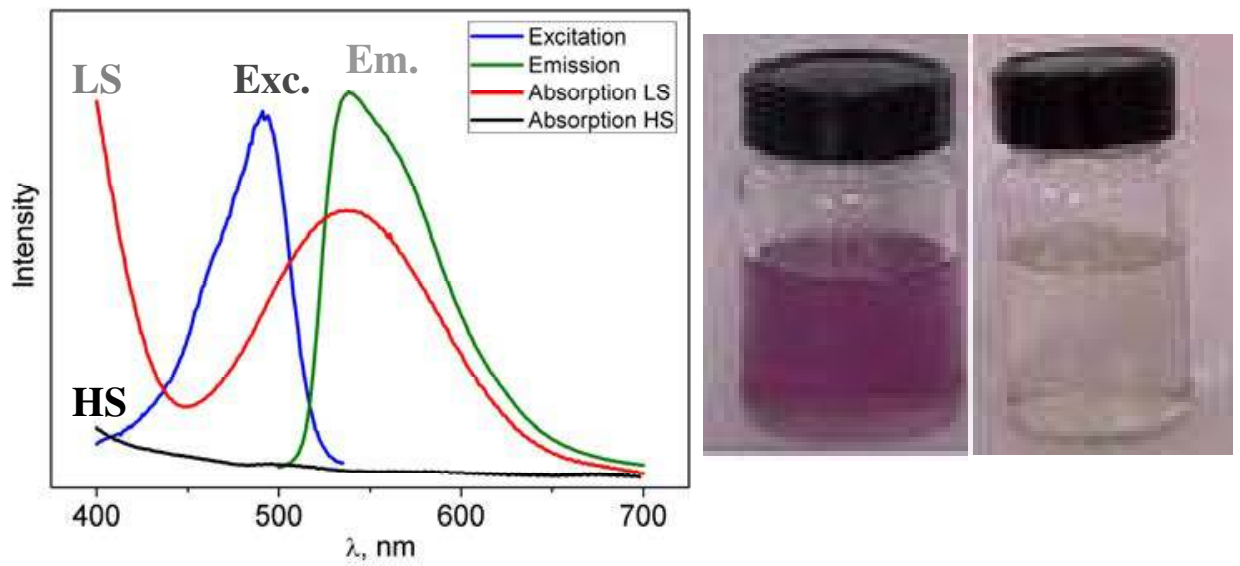

Figure 2. Optical absorption spectra of $\left[\mathrm{Fe}^{\mathrm{II}}(\mathrm{hptrz})_{3}\right](\mathrm{OTs})_{2}$ recorded in a chloroform solution at $295 \mathrm{~K}$ (LS state: red line) and at $320 \mathrm{~K}$ (HS state: black line). The photos show the color change of the solution (HS: transparent, LS: violet). Solidstate luminescence excitation (blue line) and emission (green line) spectra of $\left[\mathrm{Fe}^{\mathrm{II}}(\mathrm{hptrz})_{3}\right](\mathrm{OTs})_{2}$ doped with acridine orange. Excitation and emission spectra were recorded at $295 \mathrm{~K}$ for $560 \mathrm{~nm}$ emission and $450 \mathrm{~nm}$ excitation, respectively. Adapted from ref. [7].

Figure 3 shows the thermal variation of the luminescence intensity measured at $543 \mathrm{~nm}$ for the powder of the complex $\left[\mathrm{Fe}^{\mathrm{II}}(\mathrm{hptrz})_{3}\right](\mathrm{OTs})_{2}$ doped with the luminophore acridine orange. (Note that similar results were obtained with the luminophore Rhodamine 110 as well. ${ }^{5}$ ) This luminophore was chosen due to its robust, intense luminescence in the green spectral region. Indeed, the inspection of the absorbance spectra in figure 2 reveals that this emission overlaps tightly with the LS absorption band of the complex. At room temperature the compound is in the LS state, but upon heating it is transformed to the HS state around $325 \mathrm{~K}$. This spin state change involves the decrease of the absorbance at $543 \mathrm{~nm}$ and as a result an increase of the luminescence intensity at this wavelength. One shall note that this thermal behavior is completely opposite when compared to the ordinary thermal extinction of the luminescence. When the sample is cooled back to room temperature it returns to the LS state as seen by the variation of the luminescence intensity. The cooling and heating curves do not superpose: this thermal hysteresis is often observed in bulk SCO materials due to the cooperative nature of the spin transition. Actually, as a function of the synthesis conditions, sample composition, morphology, solvents, etc. one may tune the transition temperature of these compounds in a wide range and also the form of the transition curves (gradual, abrupt, with or without hysteresis), ${ }^{1}$ which is a very important asset for the different thermochrome applications.
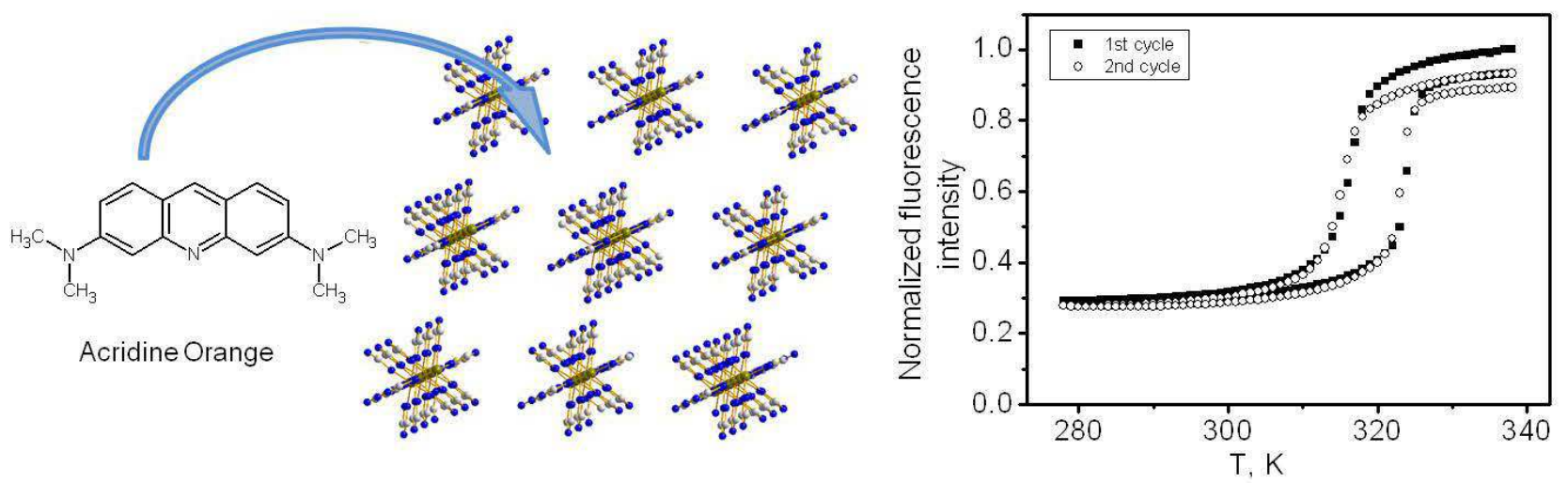

Figure 3. (a) Chemical structure of the luminophore acridine orange and schematic representation of its doping into the matrix $\left[\mathrm{Fe}^{\mathrm{II}}(\mathrm{hptrz})_{3}\right](\mathrm{OTs})_{2}$. (b) Temperature dependence of the fluorescence intensity (excitation at $450 \mathrm{~nm}$, emission at 543 $\mathrm{nm})$ of the complex $\left[\mathrm{Fe}^{\mathrm{II}}(\mathrm{hptrz})_{3}\right](\mathrm{OTs})_{2}$ doped with acridine orange $(1 \%)$ in the heating and cooling modes through two thermal cycles. 


\subsection{Sensor applications}

These assets provide a very interesting scope for using fluorescent spin crossover materials for thermometry and thermal imaging purposes. When compared to the conventional IR thermography, fluorescence thermometry can provide better sensibility (especially at low temperatures) and higher spatial resolution and may find thus applications in various fields of technology and science where accurate temperature measurements are required at reduced scales (microelectonic circuit testing, lab-on-chip devices, ....). ${ }^{19}$ The combination of luminescence with SCO is an appealing approach because it gives higher flexibility in the design of the thermometer through the separation of the sensor (SCO material) and signal transducer (fluorophore) functions. ${ }^{5-7}$ However, for practical micro-thermometry applications these hybrid systems must be processed as thin films or nano-objets. For this aim, we have developed different low-cost methods based on softlithography and spin coating. ${ }^{7}$ Figure 4 shows a proof-of-concept experiment where a spin coated thin film of the compound $\left[\mathrm{Fe}^{\mathrm{II}}(\mathrm{hptrz})_{3}\right](\mathrm{OTs})_{2}$ doped with Rhodamine 110 has been spin coated on a passivated gold microwire. Resistive heating of the wire allows for locally confined heating. With increasing temperature the spin state of the system will change locally from LS to HS and as a consequence the luminescence intensity from the heated area will increase. With precise knowledge of the relation between the luminescence intensity and the temperature, a given change in intensity can be converted to temperature. In figure 4a the bottom panel shows the fluoresecent image around the wire when no current is applied. The middle and top panels show the same measurement, but for increasing currents of $9 \mathrm{~mA}$ and $15 \mathrm{~mA}$. The strong confinement of the temperature increase in the vicinity of the wire is clearly perceptible. As shown in figure $4 \mathrm{~b}$ the measured temperature distribution profile along the wire is reasonably flat, with a steep increase of the temperature at the ends of the wire and a relatively constant value between.

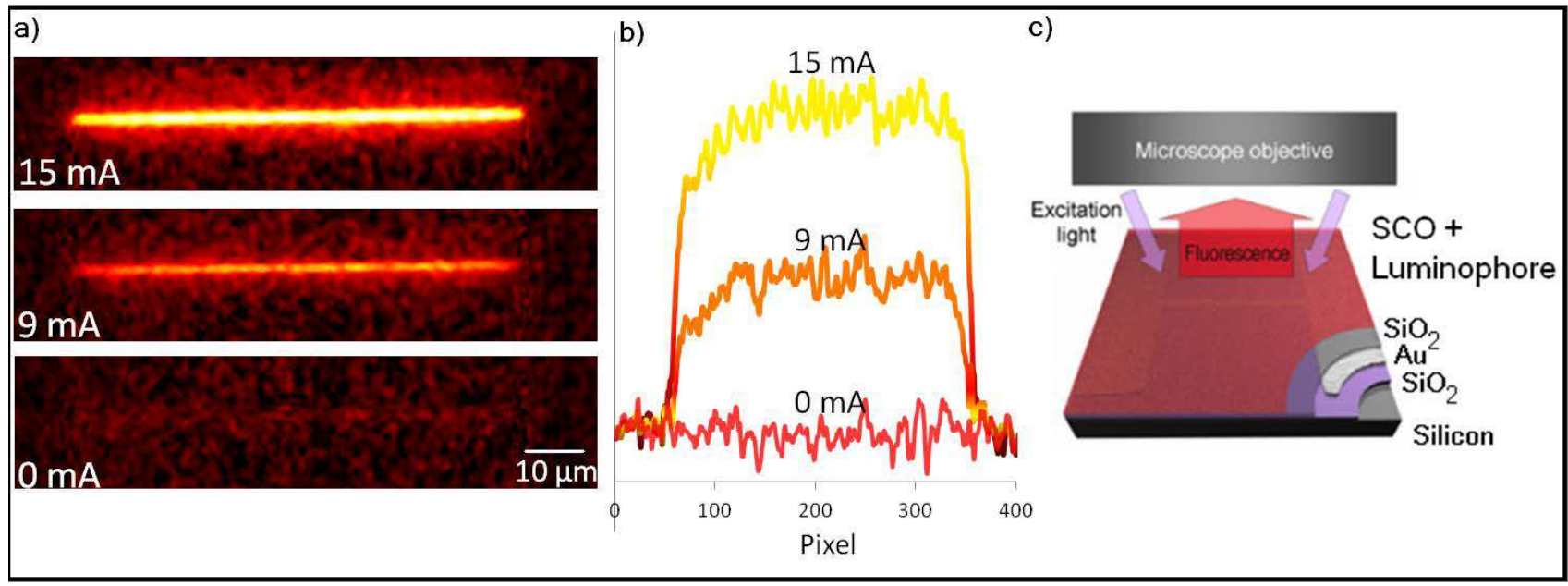

Figure 4. (a) Fluorescence microscopy images (excitation $450 \mathrm{~nm}$, emission $550 \mathrm{~nm})$ of a gold nanowire $(80 \mu \mathrm{m}$ length, $1 \mu \mathrm{m}$ width, $50 \mathrm{~nm}$ thickness) covered by a thin film of the spin crossover compound $\left[\mathrm{Fe}(\mathrm{hptrz})_{3}\right](\mathrm{OTs})_{2}$ doped with Rhodamine 110. During the fluorescence measurements the nanowires were heated by Joule effect: the current intensity is indicated for each image. (b) Cross-section of the fluorescence images along the nanowires. (c) Schematic picture of the device and the measurement setup.

Beside temperature changes several other external stimuli (application of an external pressure, light irradiation or exposure to gas/vapor molecules) can also trigger the SCO phenomenon. These stimuli can thus be used to tune the optical properties of a SCO material based device. On the other way around, the SCO phenomenon can be used to detect changes in the physicochemical environment of the compound. For example certain SCO compounds can be used in chemical sensors. ${ }^{20-21}$ To implement this idea in practical devices it may be useful to process the SCO compound as thin films or nano-patterns. ${ }^{22}$ Figure 5a shows an array of nano-objects (200 nm size, $5 \mu \mathrm{m}$ pitch) of the compound $\left[\mathrm{Fe}^{\mathrm{II}}(\mathrm{hptrz})_{3}\right](\mathrm{OTs})_{2}$ doped with Rhodamine 110 . To create this array we have developed a soft lithography approach using polydimethylsiloxane (PDMS) stamps. ${ }^{7,16}$ For patterning, a fresh chloroform solution of the complex was mixed with the luminophore and the mixture was spread onto a piece of Si wafer. Then, a nano-patterned PDMS stamp was put rapidly on top of the drop and the ensemble was heated to $80^{\circ} \mathrm{C}$ for a few minutes. As a result, nano-droplets of the solution become trapped in the cavities of the PDMS stamp and once the solvent is evaporated, they form nanodots that precipitate in an organized manner onto the Si substrate. It is known that the adsorption of ethanol vapor by the compound at room temperature results in the change of its spin state from LS to HS. Ethanol adsorption should therefore 
lead to an increase of the luminescence intensity of the nano-dots. This is shown in figure $5 \mathrm{~b}$ where the luminescence intensity of two selected dots is followed as a function of time. During the experiment the sample chamber is successively purged by dry nitrogen gas or by ethanol vapor leading to a significant decrease and increase of the luminescence signal, respectively. This type of experiment provides perspectives thus for real-time gas/vapor monitoring and even for very efficient multiplexing of signals if the nanodot compositions are different.

a)

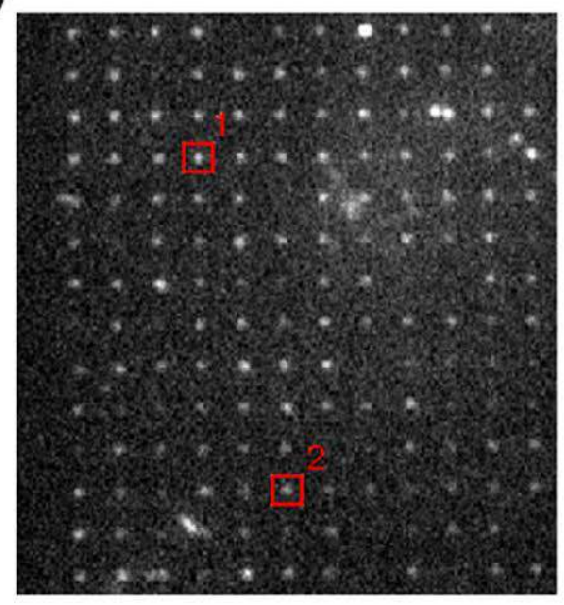

b)

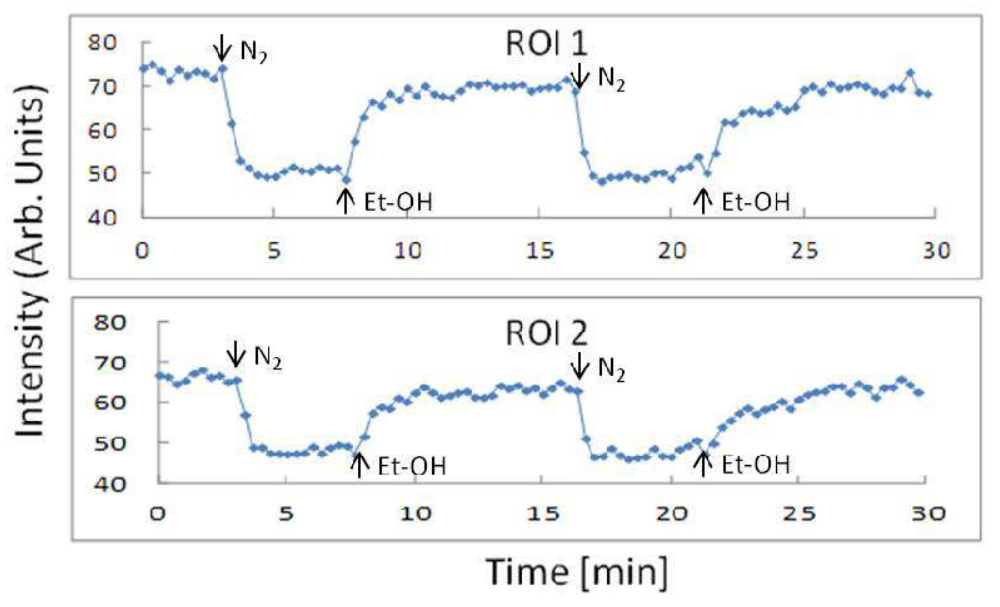

Figure 5. (a) Fluorescent microscopy image (excitation $450 \mathrm{~nm}$, emission $550 \mathrm{~nm}$ ) of an array of nano-dots $(5 \mu \mathrm{m}$ pitch) of the compound $\left[\mathrm{Fe}(\mathrm{hptrz})_{3}\right](\mathrm{OTs})_{2}$ doped by acridine orange (b) Variation of the luminescent intensity of the nano-dot $\mathrm{n}^{\circ} 1$ (top panel) and $\mathrm{n}^{\circ} 2$ (bottom panel) upon the alternate injection of ethanol vapor and dry nitrogen gas over the luminescent array at room temperature.

\section{REFRACTIVE INDEX CHANGE}

SCO materials exhibit important changes of the refractive index through the whole UV - VIS - NIR spectral ranges due primarily to the important density change accompanying the spin state change. This strong electron - lattice coupling arises from the different population of anti-bonding $e_{g}$ and non-bonding $t_{2 g}$ orbitals of the $\mathrm{Fe}^{\mathrm{II}}$ ion in the two spin states (figure 1a). The volume of the octahedron defined by the six ligating nitrogen atoms around the iron(II) ion is typically 25-30\% higher in the HS state leading to a unit cell expansion $\Delta V_{H L} / V$ between 1 and $10 \%$, depending on the nature of the compound. ${ }^{1}$ As a consequence of this significant density change, one should expect a change of the real part of the refractive index upon SCO in the range between $\Delta n_{H L}=0.01-0.1$. In addition to this material density change, one shall consider also the change of the electronic polarizability of the complex. This effect will be particularly important for wavelengths in the vicinity of intense charge transfer transitions.

The refractive index variation associated with the molecular spin state change can be exploited to construct tunable photonic devices. In such device an external stimuli allows for tuning the spin state and thus the refractive index of the active material leading to a variation of the device properties in a continuous or abrupt manner (even with hysteresis in certain cases) - depending on the nature of the SCO compound and the choice of the experimental conditions. For example spin crossover thin films can be used to modulate the propagation of electromagnetic waves in plasmonic or other type of guided wave devices. We have demonstrated this idea in ref. [17] on the example of a surface plasmon polariton (SPP) wave. Figure 6 shows the conventional attenuated total reflectance setup we used to excite SPPs at the interface of a gold layer and a thin film of the SCO complex $\left[\mathrm{Fe}^{\mathrm{II}}(\mathrm{hptrz})_{3}\right](\mathrm{OTs})_{2}$. For an appropriate combination of the wavelength, polarization and incidence angle of the exciting light beam a resonance is observed in the reflectance spectra. This is the so-called "simultaneous wavevector and frequency matching condition". The corresponding minimum in the angular reflectance curve of the Au/SCO multilayer is clearly observed in figure 6a. When the temperature of the multilayer is increased the spin state of the SCO layer changes from LS to HS and the associated decrease of the refractive index leads to a shift of the resonance to lower angles. This temperature dependent behavior occurs due to both ordinary thermal expansion of the material as well as due to the SCO phenomenon. To separate the two contributions one can plot the reflectance intensity (at a fixed angle) as a function of the temperature. As shown in figure $6 \mathrm{~b}$ the shift of the reflectance intensity is virtually linear far from the spin transition as expected for ordinary 
thermal expansion. On the other hand the discontinuity and the small hysteresis loop around $320 \mathrm{~K}$ is a consequence of the thermal spin transition. A very similar result was obtained with a diffraction grating device made of the same compound (figure 7). ${ }^{16}$ As shown in figure $7 \mathrm{c}$ the diffraction efficiency of the surface relief grating varies in a linear manner far from the spin transition, but this latter leads to an abrupt change in the diffracted light intensity. Using transmittance theory (diffraction gratings) or the Fresnel equations (SPP device), the variation of the photonic signal can be traced back quantitatively in each case to the refractive index change accompanying the SCO. ${ }^{16-17}$ These experiments highlight clearly the potential of using SCO materials for various tunable photonic devices, such as beam splitters, optical interconnects, spectral filters, antireflection coatings and polarization devices.
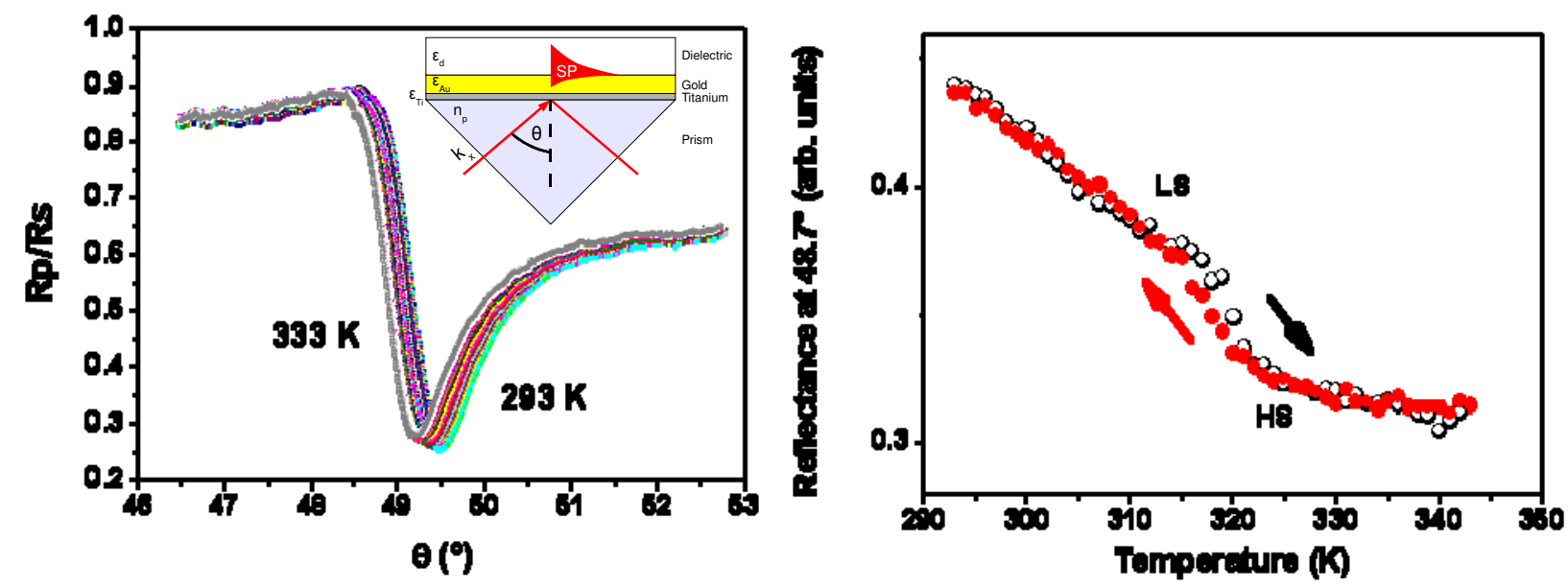

Figure 6. (a) Angle-dependent normalized reflectance spectra of a glass / Ti (5 nm) / Au (45 nm) / $\left[\mathrm{Fe}(\mathrm{hptrz})_{3}\right](\mathrm{OTs})_{2}(30$ $\mathrm{nm})$ multilayer as a function of the temperature $(\lambda=660 \mathrm{~nm})$. The inset depicts the schema of the conventional Kretchmann configuration used in the measurements. (b) Temperature dependence of the reflectance intensity (at $\theta=48.7^{\circ}$ ) of the multilayer in the heating and cooling modes $(d T / d t=2 \mathrm{~K} / \mathrm{min})$.
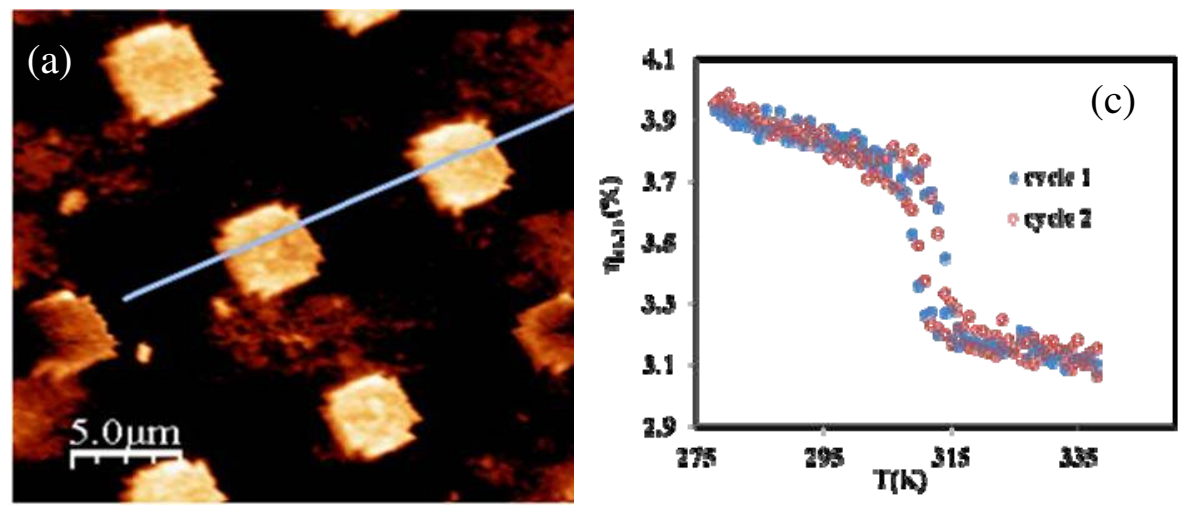

(b)
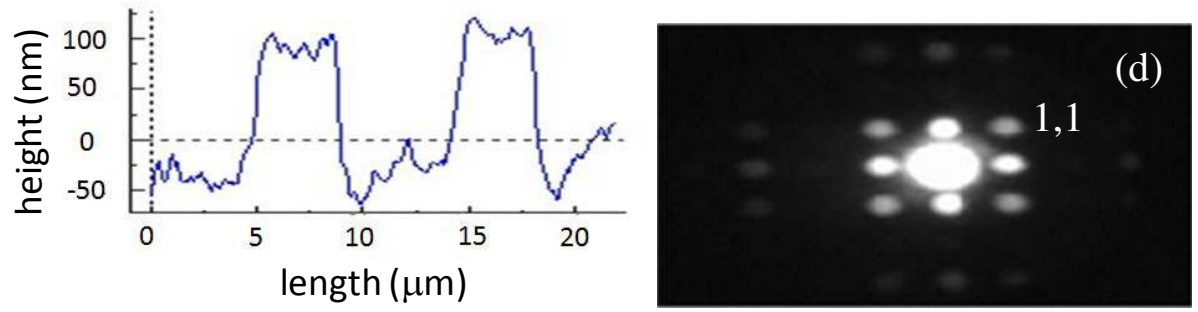

Figure 7. (a) AFM topography image and (b) height cross-section of a surface relief grating of the spin crossover compound $\left[\mathrm{Fe}(\mathrm{hptrz})_{3}\right](\mathrm{OTs})_{2}$. (c) Variation of the diffraction efficiency $(\lambda=650 \mathrm{~nm})$ for the diffracted spot $(1,1)$ as a function of the temperature through two heating - cooling cycles $(d T / d t=2 \mathrm{~K} / \mathrm{min})$. The diffraction image of the grating is shown in (d). 


\section{CONCLUSIONS AND PERSPECTIVES}

The molecular spin crossover phenomenon is accompanied by a spectacular change of the complex refractive index, including a variation of both the real and imaginary parts. With the recent advances in the synthesis of thin films and nanopatterns of these compounds it is today possible to integrate them into various photonic devices, such as fluorescence sensors, waveguides or diffraction gratings. The main interest of these photonic SCO devices comes from the fact that they respond to a variety of external stimuli and can be therefore used either to detect physicochemical changes in the environment or (vice-versa) to control the photonic properties by an external stimulus. Further work has been undertaken to explore these different possibilities and to evaluate the performance of these materials in real applications.

\section{ACKNOWLEDGMENTS}

This research was supported by the project $\operatorname{Train}^{2}$ (SUDOE) and by the "Agence National de la Recherche" through the contracts ANR-10-BLAN-1018-01 and ANR-10-NANO-012-02.

\section{REFERENCES}

[1] Gütlich, P. and Goodwin, H. A., "Spin Crossover - An Overall Perspective," Top. Curr. Chem. 233, 1-47 (2004).

[2] Kahn, O. and Martinez, C. J., "Spin-transition polymers: From molecular materials toward memory devices," Science 279, 44-48 (1998).

[3] Bousseksou, A., Salmon, L., Molnar, G. and Cobo, S., "Matériaux à transition de spin thermochromes dopés par un ou plusieurs agents fluorescents," Patent FR2952371 (13/05/2011) and WO2011058277 (19/05/2011).

[4] Engeser, M., Fabbrizzi, L., Licchelli M. and Sacchi D., "A fluorescent molecular thermometer based on the nickel(ii) high-spin/low-spin interconversion," Chem. Commun., 1191-1192 (1999).

[5] Salmon, L., Molnár, G., Zitouni, D., Quintero, C., Bergaud, C., Micheau, J.-C. and Bousseksou, A., "A novel approach for fluorescent thermometry and thermal imaging purposes using spin crossover nanoparticles," J. Mater. Chem. 20(26), 5499 - 5503 (2010).

[6] Quintero, C.M., Molnár, G., Salmon, L., Tokarev, A., Bergaud, C. and Bousseksou, A., "Design of Fluorescent Spin-Crossover Nanoparticles for Thermometry Applications," in IEEE Proceedings, $16^{\text {th }}$ International Workshop on Thermal investigations of ICs and Systems, pp. 1-5 (2010).

[7] Quintero, C.M., Gural'skiy, I.A., Salmon, L., Bergaud, C., Molnár, G. and Bousseksou, A., "Soft Lithographic Patterning of Spin Crossover Complexes. Part 1: Fluorescent Detection of the Spin Transition in Single NanoObjects," J. Mater. Chem. 22(9), 3745-3751 (2012).

[8] Garcia, Y., Robert, F., Naik, A.D., Zhou, G., Tinant, B., Robeyns, K., Michotte, S. and Piraux, L., "Spin Transition Charted in a Fluorophore-Tagged Thermochromic Dinuclear Iron(II) Complex," J. Am. Chem. Soc. 133(40), 15850-15853 (2011).

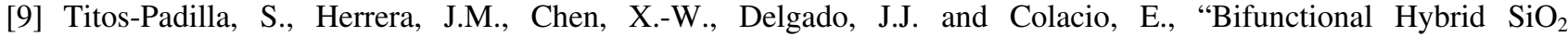
Nanoparticles Showing Synergy between Core Spin Crossover and Shell Luminescence Properties," Angew. Chem. Int. Ed. 50(14), 3290-3293 (2011).

[10] Matsuda, M., Isozaki, H. and Tajima, H., "Electroluminescence quenching caused by a spin-crossover transition," Chem. Lett. 37(3), 374-375 (2008).

[11] Hauser, A., "4-wave-mixing in the Fe(II) spin-crossover system $\left[\mathrm{Zn}_{1-X} \mathrm{Fe}_{X}(\mathrm{ptz})_{6}\right]\left(\mathrm{BF}_{4}\right)_{2}$," Chem. Phys. Lett. 202, 173-178 (1993).

[12] Loutete-Dangui, E.D., Varret, F., Codjovi, E., Dahoo, P.R., Tokoro, H., Ohkoshi, S., Eypert, C., Létard, J.-F., Coanga, J.M. and Boukheddaden, K., "Thermal spin transition in $\left[\mathrm{Fe}\left(\mathrm{NH}_{2} \mathrm{trz}\right)_{3}\right] \mathrm{Br}_{2}$ investigated by spectroscopic ellipsometry," Phys. Rev. B 75(18), 184425 (2007).

[13] Mounaix, P., Freysz, E., Degert, J., Daro, N., Létard, J.-F., Kuzel, P., Vigneras, V. and Oyenhart, L., "Dielectric characterization of $\left[\mathrm{Fe}\left(\mathrm{NH}_{2} \mathrm{trz}\right)_{3}\right] \mathrm{Br}_{2} \cdot \mathrm{H}_{2} \mathrm{O}$ thermal spin crossover compound by terahertz time domain spectroscopy," Appl. Phys. Lett. 87(24), 244103 (2005).

[14] Mounaix, P., Lascoux, N., Degert, J., Freysz, E., Kobayashi, A., Daro, N. and Létard, J.-F., "One-dimensional tunable photonic crystals with spin crossover material for the terahertz range," Appl. Phys. Lett. 89(17), 174105 (2006). 
[15] Bousseksou, A., Molnar, G., Salmon, L. and Nicolazzi, W., "Molecular Spin Crossover Phenomenon: Recent Achievements and Prospects," Chem. Soc. Rev. 40(6), 3313-3335 (2011).

[16] Akou, A., Gural'skiy, I.A., Salmon, L., Bartual-Murgui, C., Thibault, C., Vieu, C., Molnar, G. and Bousseksou, A., "Soft Lithographic Patterning of Spin Crossover Complexes. Part 2: Stimuli-Responsive Diffraction Grating Properties," J. Mater. Chem. 22(9), 3752-3757 (2012).

[17]Félix, G., Abdul-Kader, K., Mahfoud, T., Gural'skiy, I.A., Nicolazzi, W., Salmon, L., Molnar, G. and Bousseksou, A., "Surface plasmons reveal spin crossover in nanometric layers," J. Am. Chem. Soc. 133(39), 15342-15345 (2011).

[18] Hauser, A. "Ligand Field Theoretical Considerations," Top. Curr. Chem. 233, 49-58 (2004).

[19] Low, P., Kim, B., Takama, N. and Bergaud, C., "High-spatial-resolution surface-temperature mapping using fluorescent thermometry," Small 4(7), 908-914 (2008).

[20] Ohba, M., Yoneda, K., Agustí, G., Muñoz, M., Gaspar, A.B., Real, J. A., Yamasaki, M., Ando, H., Nakao, Y., Sakaki, S. and Kitagawa, S., "Bidirectional Chemo-Switching of Spin State in a Microporous Framework," Angew. Chem. Int. Ed. 48(26), 4767-4771 (2009).

[21] Agustí, G., Ohtani, R., Yoneda, K., Gaspar, A., Ohba, M., Sánchez-Royo, J., Muñoz, M., Kitagawa, S. and Real, J.A., "Oxidative Addition of Halogens on Open Metal Sites in a Microporous Spin-Crossover Coordination Polymer," Angew. Chem. Int. Ed. 48(47), 8944-8947 (2009).

[22] Bartual-Murgui, C., Akou, A., Salmon, L., Molnár, G., Thibault, C., Real, J.A. and Bousseksou, A., "Guest Effect on Nanopatterned Spin-Crossover Thin Films," Small 7(23), 3385-3391 (2011). 